\title{
POINTWISE BOUNDS ON EIGENFUNCTIONS AND WAVE PACKETS IN $N$-BODY QUANTUM SYSTEMS. I
}

\author{
BARRY SIMON ${ }^{1}$
}

\begin{abstract}
We provide a simple proof of (a modification of) Kato's theorem on the Hölder continuity of wave packets in $N$-body quantum systems. Using this method of proof and recent results of O'Connor, we prove a pointwise bound
\end{abstract}

$$
|\Psi(\zeta)| \leqq D_{\varepsilon} \exp \left[-(1-\varepsilon) a_{0}|x|\right]
$$

on discrete eigenfunctions of energy $E$. Here $\varepsilon>0, a_{0}^{2}=2$ (mass of the system) $\left[\operatorname{dist}\left(E, \sigma_{\text {ess }}\right)\right]$ and $|x|$ is the radius of gyration.

1. Introduction. In 1957, T. Kato published a beautiful paper [2] which has not received the attention it deserves. Our secondary goal in this note is to provide a simple proof of Kato's result on the Hölder continuity of "wave packets" (i.e. vectors in $C^{\infty}(H)$ ) for $N$-body quantum systems on $R^{3 N-3}$ with two body potentials. Our proof of this fact, which appears in $\S 2$, uses the basic elements of Kato's proof, especially an $L^{p}$-bootstrap; but by working in momentum space instead of configuration space, we avoid the use of modified fundamental solutions and the only $L^{p}$ estimates we will need are Hölder's and Young's inequalities.

Our interest in Kato's paper was aroused by, and our major goal is related to, recent work of $\mathrm{R}$. Ahlrichs [1] on the exponential falloff of discrete eigenfunctions of atomic systems. On physical grounds, one expects such an eigenfunction $\Psi$ to behave more or less like $\exp \left(-a_{0}|x|\right)$ as $|x| \rightarrow \infty$ where $|x|$ is the radius of gyration of the system (see $\S 3$ ) and where $a_{0}$ is a simple function of the masses of particles and the distance of the eigenvalue from the essential spectrum, (see $\S 3$ for an explicit formula). Ahlrichs proves that $\exp (a|x|) \Psi^{\top} \in L^{2}$ for any $a<a_{0}$. He then uses Kato's result to prove that $\Psi$ obeys a pointwise bound

$$
|\Psi(x)| \leqq C_{b} \exp (-b|x|)
$$

where $b<\alpha a_{0}$ with $\alpha$ an explicit constant smaller than 1 . One expects a bound of the form (1) to hold for all $b<a_{0}$ and it is this result which is our

Received by the editors December 27, 1972.

AMS (MOS) subject classifications (1970). Primary 35B40, 81A81; Secondary 35D10, 26A16, 42A12.

1 A. Sloan Fellow.

(c) American Mathematical Society 1974 
main concern here. Our proof of a pointwise bound with $b$ arbitrarily close to $a_{0}$ appears in $\S 3$.

Independently of Ahlrichs, A. O'Connor [3], [4] proved that $\exp (a|x|) \Psi \in L^{2}$ for $a<a_{0}$. O'Connor's method is very elegant, and his result is much more general than Ahlrichs requiring very minimal hypotheses on the potentials. Our proof in $\$ 3$ will result by a simple synthesis of our version of Kato's Hölder continuity theorem and O'Connor's methods.

In $\S 4$, we give a brief discussion of the extension of our results to the situation where the pair potentials are in $\boldsymbol{R}^{n}(n \neq 3)$ or where the Hamiltonian must be defined as a sum of quadratic forms [5].

It is a pleasure to thank J. Ginibre and A. O'Connor for valuable conversations and N. H. Kuiper for the hospitality of I.H.E.S.

2. Kato's Hölder continuity theorem. Throughout this section, $H_{0}$ represents an operator on $L^{2}\left(\boldsymbol{R}^{3 N-3}\right)$ of the form $H_{0}=-\sum_{i, j=1}^{3 N-3} a_{i j} \partial_{i} \partial_{j}$ where $a_{i j}$ is a positive definite matrix. We write $h(k) \equiv \sum a_{i j} k_{i} k_{j}$.

Definition. Let $2 \leqq \sigma \leqq \infty$. We say that $V$ is a potential of type $M_{\sigma}$ if $V=W+\sum_{\alpha \in I} Y_{\alpha}$ where $I$ is a finite index set and

(1) $\hat{W}$ is in $L^{1}\left(R^{3 N-3}\right)$;

(2) for each $\alpha \in I$, there is a projection $P_{\alpha}$ onto some $R^{3}$ in $R^{3 N-3}$ so that $Y_{\alpha}(x)=Z_{\alpha}\left(P_{\alpha} x\right)$ where $Z_{\alpha}$ is a function on $R^{3}$ with $Z_{\alpha} \in L^{r}+L^{1}$ where $r^{-1}+\sigma^{-1}=1$.

Remarks. (1) $\wedge$ denotes the Fourier transform.

(2) By the Hausdorff-Young inequality, $Z_{\alpha} \in L^{\sigma}+L^{\infty} \subset\left(L^{2}+L^{\infty}\right)\left(R^{3}\right)$ so $V$ is $H_{0}$-bounded with arbitrary small bound (alternately, see Lemma 1 below). Thus $H_{0}+V \equiv H$ defines a selfadjoint operator on $D\left(H_{0}\right)$.

(3) Condition $M_{\sigma}$ should be compared to Kato's condition in [2], that $W \in L^{\infty}, Z_{\alpha} \in\left(L^{\sigma}\right)_{0}$, the $L^{\sigma}$ functions of bounded support. Kato's conditions and $M_{\sigma}$ are roughly comparable, but for example if $Z_{\alpha}(x)=$ $\sin (|x|), V$ obeys Kato's conditions but not $M_{\sigma}$; if

$$
Z_{\alpha}(x)=\sum_{n=1}^{\infty} C_{n}\left|x-r_{n}\right|^{-1} \text { where } \sum\left|C_{n}\right|<\infty \text { and } r_{n} \rightarrow \infty,
$$

then $V$ obeys $M_{\sigma}$ but not Kato's conditions. In any event, either allows Yukawa or Coulomb pair interactions.

Definition. $C_{\theta}\left(\boldsymbol{R}^{n}\right)(0<\theta<1)$ denotes the uniformly Hölder continuous functions of order $\theta$, i.e. $\Psi \in C_{\theta}$ if and only if

$$
|\Psi(x)-\Psi(y)| \leqq M|x-y|^{\theta}
$$

for some $M$ and (almost) all $x, y \in R^{n}$. Similarly $\Psi \in C_{\theta}^{\prime}\left(R^{n}\right)$ means $\Psi$ is continuously differentiable and for each $i=1, \cdots, n, \partial_{i} \Psi \in C_{\theta}$. 
THEOREM 1. Let $H=H_{0}+V$ where $V$ is of type $M_{\sigma}$. Then:

(1) If $\sigma \geqq 2$, any $\Psi \in C^{\infty}(H) \equiv \bigcap_{m} D\left(H^{m}\right)$ is in $C_{\theta}\left(\boldsymbol{R}^{3 N-3}\right)$ for any $\theta<\min \left(1,2-3 \sigma^{-1}\right)$.

(2) If $\sigma>3$, any $\Psi \in C^{\infty}(H)$ is in $C_{\theta}^{\prime}\left(\boldsymbol{R}^{2 N-3}\right)$ for any $\theta<1-3 \sigma^{-1}$.

Remarks. (1) As we shall see, the condition $\Psi \in C^{\infty}(H)$ can be replaced with $\Psi \in D\left(H^{m}\right)$ for some $m$ with $(m-1)\left(4-6 \sigma^{-1}\right)>3 N-3$.

(2) If $C^{\infty}(H)$ is topologized with the norms $\|\Psi\|_{m}=\left\|H^{m} \Psi\right\|$ and if $C_{\theta}$ (resp. $C_{\theta}^{\prime}$ ) is topologized with the norm

$$
\|f\|_{\theta}=\sup _{x}|f(x)|+\sup _{x, y}\left[|x-y|^{-\theta}|f(x)-f(y)|\right]
$$

(resp. $\|f\|_{\theta}^{\prime}=\sup _{x}|f(x)|+\sum_{j=1}^{n}\left\|\partial_{j} f\right\|_{\theta}$ ), then the imbeddings $C^{\infty}(H) \subset C_{\theta}$ guaranteed by the theorem are continuous.

(3) Except for a slight difference in the assumptions on $V$, this is the main theorem (Theorem I) of [2].

(4) The basic perturbation estimate tells us that $\left(H_{0}+I\right)^{-1} V$ is bounded from $L^{2}$ to $L^{2}$. Our proof (like Kato's) is based on two ways in which this can be improved. First $\left(H_{0}+I\right)^{-\beta} V$ is bounded for certain $\beta<1$ and secondly it is bounded on certain $L^{p}$ spaces.

LeMma 1. Let $V$ be of type $M_{\sigma}$ and let $\beta>3 / 2 \sigma$. Suppose that $1 \leqq p \leqq 2$ and let $\hat{\Psi} \in L^{p}$. Then $\left(\left(H_{0}+I\right)^{-\beta} V \Psi\right)^{\wedge} \in L^{p}$.

REMARK. This lemma (and similar statements later) are intended to hold in the sense of a priori estimates

for all $\Psi \in \mathscr{S}\left(\boldsymbol{R}^{3 N-3}\right)$.

$$
\left\|\left(\left(H_{0}+I\right)^{-\beta} V \Psi\right)\right\|_{p} \leqq C\|\hat{\Psi}\|_{p}
$$

PROOF.

$$
\left(\left(H_{0}+I\right)^{-\beta} V \Psi\right)^{\wedge}=(2 \pi)^{(3 . N-3) / 2}(h(k)+1)^{-\beta} \hat{V} * \hat{\Psi} .
$$

We consider the individual terms $\hat{W} * \hat{\Psi}$ and $\hat{Y}_{\alpha} * \hat{\Psi}$ in $\hat{V} * \hat{\Psi}$. Since $\hat{W} \in L^{1}$ and $(h(k)+1)^{-\beta} \in L^{\infty}$,

$$
\left\|(h(k)+1)^{-\beta} \hat{W} * \hat{\Psi}\right\|_{p} \leqq\left\|(h(k)+1)^{-\beta}\right\|_{\infty}\|\hat{W}\|_{1}\|\hat{\Psi}\|_{p}
$$

by Young's and Hölder's inequalities.

Write $k_{\alpha}$ for the 3 coordinates in $\operatorname{Ran} P_{\alpha}$ and $k_{\alpha}^{\perp}$ for $3 N-6$ orthogonal coordinates. Since $\left(k_{x}^{2}+1\right)^{-\beta} \in L^{\sigma}\left(\boldsymbol{R}^{3}\right)$ for each $p \leqq \sigma$,

$$
\left\|\left(k_{\alpha}^{2}+1\right)^{-\beta}\left(\hat{Z}_{\alpha} * f\right)\left(k_{\alpha}\right)\right\|_{p} \leqq C\|f\|_{\nu} .
$$

Thus for each $p \leqq 2$ and each fixed $k_{x}^{\perp}$ :

$$
\int\left|\left(k_{x}^{2}+1\right)^{-\beta} \int Z_{x}\left(k_{x}-k_{x}^{\prime}\right) f\left(k_{\alpha}^{\prime}, k_{\alpha}^{1}\right) d k_{\alpha}^{\prime}\right|^{p} d k_{x} \leqq\left. C \int|f|\left(k_{\alpha}, k_{\alpha}^{1}\right)\right|^{p} d k_{\alpha} .
$$


Integrating over $k_{\alpha}^{\perp}$, we conclude that $\left\|\left(k_{\alpha}^{2}+1\right)^{-\beta} \hat{Y}_{\alpha} * \hat{\Psi}\right\|_{p} \leqq C_{1}\|\hat{\Psi}\|_{p}$. Since $\left(k_{\alpha}^{2}+1\right)^{\beta}(h(k)+1)^{-\beta} \in L^{\infty}\left(\boldsymbol{R}^{3 N-3}\right)$, the lemma follows.

LEMMA 2. Let $V$ be of type $M_{\sigma}$ and let $\gamma<1-3 / 2 \sigma$. Let $1 \leqq p \leqq 2$. If $\hat{\Psi},(H \Psi)^{\wedge} \in L^{p}$, then $\left(1+|k|^{2}\right)^{\gamma} \hat{\Psi}^{\sigma} \in L^{p}$.

Proof. Since $(H+1) \Psi=\left(H_{0}+1\right) \Psi+V \Psi$, we have $\Psi=\left(H_{0}+1\right)^{-1} \times$ $(H+1) \Psi-\left(H_{0}+1\right)^{-1} V \Psi$. So:

$$
\begin{aligned}
\left(1+|k|^{2}\right)^{\gamma} \hat{\Psi}= & \left(1+|k|^{2}\right)^{\gamma-1}\left[\left(1+|k|^{2}\right) /(1+h(k)]\left((H+1)^{4}\right)^{\wedge}\right. \\
& -\left[\left(1+|k|^{2}\right) /(1+h(k))\right]^{\gamma}\left(\left(H_{0}+1\right)^{-\beta} V \Psi\right)^{\wedge}
\end{aligned}
$$

where $\beta=1-\gamma>3 / 2 \sigma$. By hypothesis, the first term on the right-hand side of (2) is in $L^{p}$ and by Lemma 1 , the second term is in $L^{p}$.

For the reader's convenience, we include the following standard result:

LEMMA 3. If $\left(1+|k|^{2}\right)^{\gamma} \hat{\Psi} \in L^{1}\left(\boldsymbol{R}^{n}\right)$ for $\gamma>0$, then $\Psi$ is $C_{\theta}$ for any $\theta$ with $\theta<\min (1,2 \gamma)$. If $\gamma>\frac{1}{2}$, then $\Psi$ is $C_{\theta}^{\prime}$ for any $\theta<\min (1,2 \gamma-1)$.

Proof. For any $y \in R,\left|e^{i y}-1\right| \leqq 2$ and $\left|e^{i y}-1\right|=\left|\int_{0}^{y} e^{i x} d x\right| \leqq y$. Therefore, for any $\theta \leqq 1$ and all $k, x$ and $y \in \boldsymbol{R}^{n},\left|e^{i k \cdot y}-e^{i k \cdot y}\right| \leqq$ $2^{(1-\theta)}|k|^{\theta}|x-y|^{\theta}$. Thus:

$|\Psi(x)-\Psi(y)|$

$$
\leqq(2 \pi)^{-n / 2} 2^{1-\theta}|x-y|^{\theta}\left\||k|^{\theta}\left(1+|k|^{2}\right)^{-\gamma}\right\|_{\infty}\left\|\left(1+|k|^{2}\right)^{\gamma} \hat{\Psi}\right\|_{1} .
$$

This proves the first statement in the lemma. The second has a similar proof using

$$
\left|e^{i y}-1-i y\right| \leqq 2|y| \text { and }\left|e^{i y}-1-i y\right| \leqq \frac{1}{2}|y|^{2} .
$$

Proof OF THEOREM 1. Since $\left(1+|k|^{2}\right)^{-\gamma} \in L^{q}\left(\boldsymbol{R}^{n}\right)$ for all $q>n / 2 \gamma$, Lemma 2 implies that if $\hat{\Psi},(H \Psi)^{\wedge} \in L^{p}$, then $\hat{\Psi} \in L^{r}$ for all $r \geqq 1$ obeying $r \geqq\left(p^{-1}+(2 \gamma /(3 N-3))\right)^{-1}$. By induction if $m \geqq k$ and if $\hat{\Psi}, \cdots,\left(H^{m} \Psi\right)^{\wedge} \in$ $L^{2}$ then $\hat{\Psi}^{*}, \cdots,\left(H^{k} \Psi\right)^{\wedge} \in L^{r}$ if $r \geqq 1$ and $r \geqq\left(\frac{1}{2}+(m-k)(2 \gamma /(3 N-3))\right)^{-1}$. Since $\gamma$ can be chosen arbitrarily close to $1-3 / 2 \sigma$, we have that for any integer $m$ with $(2 m)(2-3 / \sigma)>3 N-3$,

(i) $\Psi \in D\left(H^{m}\right)$ implies $\hat{\Psi} \in L^{1}$;

(ii) $\Psi \in D\left(H^{m+1}\right)$ implies that $\left(1+|k|^{2}\right)^{\gamma} \hat{\Psi} \in L^{1}$ if $\gamma<1-3 / 2 \sigma$.

Lemma 3 completes the proof.

3. Pointwise exponential falloff of discrete eigenfunctions. By an $N$-body quantum Hamiltonian of type $M_{\sigma}$, we will mean an operator $\tilde{H}$ on $L^{2}\left(\boldsymbol{R}^{3 N}\right)$ of the form

$$
\tilde{H}=-\sum_{i=1}^{N}\left(2 m_{i}\right)^{-1} \Delta_{i}+\sum_{i<j=1}^{N} V_{i j}\left(\boldsymbol{r}_{i}-\boldsymbol{r}_{j}\right) .
$$


Where a point in $\boldsymbol{R}^{3 N}$ is written $\left(\boldsymbol{r}_{i}, \cdots, \boldsymbol{r}_{N}\right)$ with $\boldsymbol{r}_{i} \in \boldsymbol{R}^{3}, \Delta_{i}$ is the Laplacian with respect to $r_{i}$ and $V_{i j}$ is a function on $R^{3}$ with $\hat{V}_{i j} \in L^{a}+L^{1}$ where $q^{-1}+\sigma^{-1}=1$.

Write $M=\sum_{i=1}^{N} m_{i}$ (total mass), $\mathbf{R}=M^{-1} \sum_{i=1}^{N} m_{i} r_{i}$ (center of mass) and

$$
x=\left(\sum_{i=1}^{N} m_{i} M^{-1}\left|r_{i}-\mathbf{R}\right|^{2}\right)^{1 / 2}
$$

(radius of gyration). In a standard way we can choose linear coordinates $\left(\zeta_{1}, \cdots, \zeta_{N-1}, R\right)$ so that under the resulting decomposition

$$
\begin{aligned}
& L^{2}\left(R^{3 N}\right)=L^{2}\left(R^{3 N-3}\right) \otimes L^{2}\left(R^{3}\right), \\
& \tilde{H}=H \otimes 1+1 \otimes(2 M)^{-1} \Delta .
\end{aligned}
$$

We will call $H$ a reduced $N$-body quantum Hamiltonian of type $M_{\sigma}$. Such a Hamiltonian is always of the form $H_{0}+V$ where $V$ is a potential of type $M_{\sigma}$ in the sense of $\$ 2$. By a further linear coordinate change (of Jacobian not necessarily 1), we can suppose that $x^{2}=\sum_{i=1}^{N-1}\left|\zeta_{i}\right|^{2}$ in which case

$$
H_{0}=(-2 M)^{-1} \sum_{i=1}^{N-1} \Delta_{\zeta_{i}} .
$$

THEOREM 2. Let $H$ be a reduced $N$-body quantum Hamiltonian of type $M_{\sigma}$. Let $E_{c}=\inf \sigma_{\text {ess }}(H)$ and suppose that $H \Psi=E \Psi$ with $E<E_{c}$. Let $a_{0}=\left(2 M\left(E_{c}-E\right)\right)^{1 / 2}$ and let $|x|$ be the radius of gyration. Then

(1) For any $a_{1}<a_{0}$, there exists a constant $D_{a_{1}}$ with

for all $\zeta \in \boldsymbol{R}^{3 N-3}$.

$$
|\Psi(\zeta)| \leqq D_{a_{1}} \exp \left(-a_{1}|x|\right)
$$

(2) For any $a_{1}<a_{0}$, and $\theta<\min \left(1,2-3 \sigma^{-1}\right)$, there exists a constant $D_{\theta, a}$ with for all $\zeta, \zeta^{\prime} \in \boldsymbol{R}^{3 N-3}$.

$$
\left|\Psi(\zeta)-\Psi\left(\zeta^{\prime}\right)\right| \leqq D_{\theta, a_{1}} \exp \left[-a_{1} \min \left(|\zeta|,\left|\zeta^{\prime}\right|\right)\right]\left|\zeta-\zeta^{\prime}\right|^{\theta}
$$

(3) If $\sigma>3$, for any $a_{1}<a_{0}$, and $\theta<1-3 \sigma^{-1}$, there exists $D_{\theta, a_{1}}^{\prime}$ with

$\left|\operatorname{grad} \Psi(\zeta)-\operatorname{grad} \Psi\left(\zeta^{\prime}\right)\right| \leqq D_{\theta, a_{1}}^{\prime} \exp \left[-a_{1} \min \left(|\zeta|,\left|\zeta^{\prime}\right|\right)\right]\left|\zeta-\zeta^{\prime}\right|^{\theta}$ for all $\zeta, \zeta^{\prime} \in R^{3 N-3}$.

REMARK. The constants, $D_{a_{1}}, D_{\theta, a_{1}}$ and $D_{\theta, a_{1}}^{\prime}$ depend on $V$ only through $L^{p}$ norms of the $\hat{V}_{i j}$.

Proof. Suppose $H$ is in normal form. By a Payley-Wiener argument (see, e.g. O'Connor [3], [4]), we need only prove that $\hat{\Psi}$ has an analytic continuation to the tube $\left\{k \in C^{3 N-3}|| \operatorname{Im} k \mid<a_{0}\right\}$ so that if $\hat{\Psi}_{a}$ is defined by $\hat{\Psi}_{a}(k)=\hat{\Psi}(k+i a)$ for any $a \in R^{3 N-3}$ with $|a|<a_{0}$, then $\left(1+k^{2}\right)^{\gamma} \hat{\Psi}_{a} \in L^{1}$ 
with $L^{1}$ norm bounded as $a$ runs through the set $\left\{a|| a \mid<a_{1}\right\}$ for each $a_{1}<a_{0}$. Here $\gamma$ is any real less than $1-3 / 2 \sigma$.

O'Connor [3], [4] has already proven that such a continuation exists with $\hat{\Psi}_{a} \in L^{2}$ uniformly as $a$ runs through sets of the form $\left\{a|| a \mid<a_{1}\right\}$. Moreover $\hat{\Psi}_{a}$ obeys the equation

$$
\left((k+i a)^{2}-E\right) \hat{\Psi}_{a}=(2 \pi)^{(3 N-3) / 2} \hat{V} * \hat{\Psi}_{a}
$$

By mimicking our argument in $\S 2$, the equation (3), the condition that $V$ be of type $M_{\sigma}$ and O'Connor's $L^{2}$ bounds imply the required $L^{1}$ bound on $\left(1+k^{2}\right)^{\gamma} \hat{\Psi}^{\circ}$.

4. Extension to higher dimensions and to operators defined by quadratic forms. In this section, we wish to generalize Theorem 1; a similar generalization of Theorem 2 holds. Since there are few new ideas, we only sketch the arguments.

Definition. Let $\sigma \geqq 1$. We say that $V$ is a potential of type $M_{\sigma}^{(m)}$ on $\boldsymbol{R}^{m N-m}$ if $V=W+\sum_{\alpha \in I} Y_{\alpha}$ where $I$ is a finite index set and if

(1) $\hat{W} \in L^{1}\left(\boldsymbol{R}^{m N-m}\right)$.

(2) For each $\alpha \in I$, there is a projection $P_{\alpha}$ onto an $\boldsymbol{R}^{m}$ in $\boldsymbol{R}^{m N-m}$ and a function $Z_{\alpha}$ on $\boldsymbol{R}^{m}$ with $\hat{Z}_{\alpha} \in L^{r}+L^{1}\left(r^{-1}+\sigma^{-1}=1\right)$ so that $Y_{\alpha}(x)=$ $Z_{\alpha}\left(P_{\alpha} x\right)$.

If $\sigma \geqq 2$ and $\sigma>m / 2$, then $H_{0}+V$ can be defined as a selfadjoint operator sum. If $2>\sigma>m / 2$ (in particular, only when $m \leqq 3$ ), we can define $H_{0}+V$ as a selfadjoint operator which is the sum of $H_{0}$ and $V$ as quadratic forms [5]. We have:

THEOREM $1^{\prime}$. Let $H=H_{0}+V$ where $V$ is of type $M_{\sigma}^{(m)}$ with $\sigma>m / 2$ (and $\sigma \geqq 1$ ). Then:

(1) Any $\Psi \in C^{\infty}(H)$ is in $C_{\theta}\left(\boldsymbol{R}^{m N-m}\right)$ for any $\theta<\min \left(1,2-m \sigma^{-1}\right)$.

(2) If $\sigma>m$, any $\Psi \in C^{\infty}(H)$ is in $C_{\theta}^{\prime}\left(\boldsymbol{R}^{m N^{\prime}-m}\right)$ for any $\theta<1-m \sigma^{-1}$.

Sketch of Proof. Case 1: $\sigma>2$. Our proof of Theorem 1 goes through with minor modifications; Lemma 1 holds if $\beta>m / 2 \sigma$ and Lemma 2 if $\gamma<1-m / 2 \sigma$. The condition $\sigma \geqq 2$ enters in the proof of Lemma 1 , since to apply Young's inequality to $L^{p} * L^{q}$ we need $p^{-1}+q^{-1}>1$.

Case 2: $2 \geqq \sigma>m / 2$. A simple quadratic form modification. We first note that Lemma 1 holds if $\beta>m / 2 \sigma$ and if $p \leqq \sigma$. Moreover, we have:

LEMma $1^{\prime}$. Let $\sigma \leqq p \leqq 2$ and define $\alpha$ by $\alpha^{-1}+\sigma / p=1$. Let $\beta>m / 2 \sigma$ and let $\hat{\Psi} \in L^{p}$. Then the Fourier transform of $\left(H_{0}+1\right)^{-(1-\alpha) \beta} V\left(H_{0}+1\right)^{-\alpha \beta} \Psi$ is in $L^{p}$.

LEMMA $2^{\prime}$. Let $p, \sigma, \alpha, \beta$ be as in Lemma $1^{\prime}$. Suppose that $\left(1+k^{2}\right)^{\alpha \beta} \hat{\Psi}$, $(H \Psi)^{\wedge} \in L^{p}$. Let $\gamma<1-m / 2 \sigma$. Then $\left(1+k^{2}\right)^{\gamma}\left(1+k^{2}\right)^{\alpha \beta} \hat{\Psi} \in L^{p}$. 
The proofs of Lemmas $1^{\prime}$ and $2^{\prime}$ follow the pattern of Lemmas 1 and 2. If $\Psi \in C^{\infty}(H)$, then $H^{n} \Psi \in Q(H)$, the form domain of $H$ for each $n$. Since $Q(H)=Q\left(H_{0}\right),\left(1+k^{2}\right)^{1 / 2}\left(H^{n} \Psi\right)^{\wedge} \in L^{2}$ for all $n$. By a finite induction using Lemma $2^{\prime},\left(H^{n} \Psi\right)^{\wedge} \in L^{\sigma}$. Lemma 1 is now applicable and the proof is completed as in Theorem 1.

One can ask if some modified version of Theorem $1^{\prime}$ remains true at the borderline value $\sigma=m / 2$. If $m \geqq 5, H_{0}+V$ can be defined as an operator sum if $V$ is of type $M_{m / 2}^{(m)}$ and if $m=2,3,4, H_{0}+V$ can be defined as a sum of forms. However, in this borderline case, there may be unbounded functions $\Psi \in C^{\infty}(H)$.

EXAMPLE. Let $m \geqq 3$ and let $\Psi$ be a spherically symmetric function on $\boldsymbol{R}^{m}$ so that (i) $\Psi$ is $C^{\infty}$ and strictly positive on $\boldsymbol{R}^{m} \backslash\{0\}$. (ii) In the region $R_{1}=\{x|| x \mid \geqq 1\} \Psi$ obeys $-\Delta \Psi=-\Psi$ and $\Psi \rightarrow 0$ as $|x| \rightarrow \infty$. (iii) In the region $R_{2}=\left\{x|| x \mid \leqq \frac{1}{2}\right\}, \Psi(x)=-\ln |x|$. It is easy to construct such a function. Let $V(x)=-1+(\Delta \Psi / \Psi)$. Then $V$ has support in $R^{m} \backslash R_{1}$, and in the region $R_{2}, V(x)=-1+C_{m} r^{-2}(\ln r)^{-1}$. Thus $V \in L^{m / 2}$ (and in particular, if $m=3, V \in R$, the Rollnik class [5]) and $\Psi$ is in $C^{\infty}(H)$ and is unbounded.

REMARK. The above example does not work in case $m=2$, because $-\Delta(\ln r)=C_{2} \delta(x)$; but if we modify $\Psi$ to equal $(-\ln |x|)^{\alpha}$ with $0<\alpha<1$ in $R_{2}$, then $V=-1+d_{\alpha} r^{-2}(\ln r)^{-2}$ in $R_{2}$ so $V \in L^{1}\left(R^{2}\right)$. Thus there is a borderline example in $\boldsymbol{R}^{2}$.

\section{REFERENCES}

1. R. Ahlrichs, Asymptotic behavior of atomic bound state wave functions, Univ. of Karlsruhe, April 1972 (preprint).

2. T. Kato, On the eigenfunctions of many-particle systems in quantum mechanics, Comm. Pure Appl. Math. 10 (1957), 151-177. MR 19, 501.

3. A. O'Connor, Thesis, Princeton University, Princeton, N.J., 1972.

4. - Exponential decay of bound state wave functions, Comm. Math. Phys. (to appear).

5. B. Simon, Quantum mechanics for Hamiltonians defined as quadratic forms, Princeton Univ. Press, Princeton, N.J., 1971.

I.H.E.S., BURES-SUR-YvetTe, FRANCE

Current address: Department(s) of Mathematics and Physics, Princeton University, Princeton, New Jersey 08540 